NT@UW-01-016

\title{
The Magnetic Moments of the Octet Baryons in Quenched Chiral Perturbation Theory
}

\author{
Martin J. Savage* \\ Department of Physics, University of Washington, Seattle, WA 98195-1560.
}

\begin{abstract}
We compute the magnetic moments of the octet baryons up to two orders in quenched chiral perturbation theory. In addition to the $\sim \sqrt{m_{q}}$ contributions that arise in QCD, there are lower-order contributions of the form $M_{0}^{2} \log m_{q}$ from loop diagrams involving hairpin interactions.
\end{abstract}

July 2001

*savage@phys . washington. edu 


\section{INTRODUCTION}

The magnetic moments of the octet-baryons continue to play a crucial role in our understanding of hadronic structure from the underlying theory of strong interactions, QCD, and also in the context of more phenomenological models, such as the non-relativistic quark model (NRQM). Perhaps one of the most intriguing aspects of strong interaction phenomenology is the remarkable success enjoyed by naive NRQM's in describing the magnetic moments of the low-lying baryons based on the simple picture of weakly interacting Dirac particles with masses tuned to the baryon mass spectrum and one-third integer charges. Clearly, one wishes to understand this success directly from QCD but at this point in time such illuminating calculations do not exist.

In the future, numerical lattice gauge calculations of many hadronic observables, including the magnetic moments, will be performed. However, at present and in the near future, lattice calculations cannot be performed with the light quark masses, $m_{q}$, near their physical values $\left(m_{u} \sim 5 \mathrm{MeV}, m_{d} \sim 10 \mathrm{MeV}\right.$ and $\left.m_{s} \sim 125 \mathrm{MeV}\right)$ and extrapolation from the lattice quark masses $\left(m_{\pi}^{\text {latt. }} \sim 500 \mathrm{MeV}\right)$ is required. First efforts at extrapolating quenched lattice evaluations of the octet magnetic moments have been made in Refs. [1]. Quenched lattice data [2] was used in conjunction with a Pade approximant to extrapolate from $m_{\pi}^{\text {latt. }} \gtrsim 600 \mathrm{MeV}$ to the physical value of the pion mass. Although the Pade approximant cannot be rigorously justified, it does reproduce the correct small $m_{q}$ behavior [3] $\left(\sim \sqrt{m_{q}}\right)$ of the magnetic moments in QCD obtained from chiral perturbation theory, and also the decoupling expected for large $m_{\pi}$, by construction. This phenomenological extrapolation of the quenched data compares reasonably well with the measured values of the magnetic moments for many of the octet baryons, however, there are still significant discrepancies that remain. One avenue that could be explored is to include the higher order $m_{q}$ dependences that have been computed during the past several years [4] into the Pade approximant. However, given that quenched QCD (QQCD) is not QCD, and that the Pade approximant cannot be justified from QCD it is perhaps more interesting to know the predictions of QQCD itself for the magnetic moments, and not to attempt to match QQCD to the real world. Such predictions would require calculations in QQCD with quark masses that are small enough to match onto the chiral expansion and also a calculation of the magnetic moments in quenched chiral perturbation theory $(\mathrm{Q} \chi \mathrm{PT})$ [8], which is what we will do here.

In QCD the expansion of a baryon magnetic moment $\mu_{B}$ about the chiral limit has the form $\mu_{B} \sim \mu_{0}+\beta \sqrt{m_{q}}+\gamma m_{q} \log m_{q}+\ldots$, where $\mu_{0}$ is a combination of the two coefficients of local dimension- 5 operators that contribute at leading order in the chiral expansion. However, in QQCD the presence of the light $\eta^{\prime}$, and the associated "hairpin" interactions render QQCD more divergent in the infrared than QCD. Therefore, in QQCD the chiral expansion for the magnetic moments of the low-lying baryons is of the form

$\mu_{B}^{\mathrm{QQCD}} \sim \mu_{0}^{\mathrm{QQCD}}+\delta \log m_{q}+\beta^{\mathrm{QQCD}} \sqrt{m_{q}}+\ldots$, where $\mu_{0}^{\mathrm{QQCD}} \neq \mu_{0}$, and $\beta^{\mathrm{QQCD}} \neq \beta$. Further, the coefficient $\delta$ is entirely an artifact of quenching. In this work we compute the magnetic moments of the octet-baryons up to two orders in $\mathrm{Q} \chi \mathrm{PT}$, that is we compute $\delta$ and $\beta^{\mathrm{QQCD}}$ for each baryon.

$\mathrm{Q} \chi \mathrm{PT}$ for the low-lying baryons has been set-up in a pioneering paper by Labrenz and Sharpe (LS) [1] and used to compute the chiral corrections to the octet and decuplet baryon 
masses. LS used quark-line diagrams to identify and compute the meson-loop diagrams that contribute. In this work, we will use ghost-baryon fields to compute the one-loop diagrams that contribute to the magnetic moments, in a way similar to that used to compute properties of baryons containing heavy quarks [12].

\section{II. $\mathbf{Q} \chi \mathbf{P T}$}

The lagrange density of QQCD is

$$
\begin{aligned}
\mathcal{L} & =\sum_{a, b=u, d, s} \bar{q}^{a}\left[i \not D-m_{q}\right]_{a}^{b} q_{b}+\sum_{\tilde{a}, \tilde{b}=\tilde{u}, \tilde{d}, \tilde{s}} \overline{\tilde{q}}^{\tilde{a}}\left[i \not D-m_{\tilde{q}}\right]_{\tilde{a}}^{\tilde{b}} \tilde{q}_{\tilde{b}} \\
& =\sum_{j, k=u, d, s, \tilde{u}, \tilde{d}, \tilde{s}} \bar{Q}^{j}\left[i \not D-m_{Q}\right]_{j}^{k} Q_{k},
\end{aligned}
$$

where $q_{a}$ are the three light-quarks, $u, d$, and $s$, and $\tilde{q}$ are three light bosonic quarks $\tilde{u}, \tilde{d}$, and $\tilde{s}$. The super-quark field, $Q_{j}$, is a six-component column vector with the three light-quarks, $u, d$, and $s$, in the upper three entries and the three ghost-light-quarks, $\tilde{u}, \tilde{d}$, and $\tilde{s}$, in the lower three entries. The graded equal-time commutation relations for two fields is

$$
Q_{i}^{\alpha}(\mathbf{x}) Q_{j}^{\beta \dagger}(\mathbf{y})-(-)^{\eta_{i} \eta_{j}} Q_{j}^{\beta \dagger}(\mathbf{y}) Q_{i}^{\alpha}(\mathbf{x})=\delta^{\alpha \beta} \delta_{i j} \delta^{3}(\mathbf{x}-\mathbf{y})
$$

where $\alpha, \beta$ are Dirac-indices and $i, j$ are flavor indices. The objects $\eta_{k}$ correspond to the parity of the component of $Q_{k}$, with $\eta_{k}=+1$ for $k=1,2,3$ and $\eta_{k}=0$ for $k=4,5,6$, and the graded commutation relations for two $Q$ 's or two $Q^{\dagger}$ 's are analogous. The diagonal super mass-matrix, $m_{Q}$, has entries $m_{Q}=\operatorname{diag}\left(m_{u}, m_{d}, m_{s}, m_{u}, m_{d}, m_{s}\right)$, i.e. $m_{\tilde{u}}=m_{u}, m_{\tilde{d}}=m_{d}$ and $m_{\tilde{s}}=m_{s}$, so that the contribution to the determinant in the path integral from the $q$ 's and the $\tilde{q}$ 's exactly cancel.

In the absence of quark masses, the lagrange density in eq. (1) has a graded symmetry $U(3 \mid 3)_{L} \otimes U(3 \mid 3)_{R}$, where the left- and right-handed quark fields transform as $Q_{L} \rightarrow U_{L} Q_{L}$ and $Q_{R} \rightarrow U_{R} Q_{R}$ respectively. However, the functional integral associated with this Lagrange density does not converge unless the transformations on the left- and right-handed fields are related, $\operatorname{sdet}\left(U_{L}\right)=\operatorname{sdet}\left(U_{R}\right)$, where $\operatorname{sdet}()$ denotes a superdeterminant [9,10,13,14], leaving the theory to have a symmetry $\left[S U(3 \mid 3)_{L} \otimes S U(3 \mid 3)_{R}\right] \times U(1)_{V}$, where the " $x$ " denotes a semi-direct product as opposed to a direct product, " $\otimes$ " $\mathrm{q}$. It is assumed that this symmetry is spontaneously broken $\left[S U(3 \mid 3)_{L} \otimes S U(3 \mid 3)_{R}\right] \times U(1)_{V} \rightarrow$ $S U(3 \mid 3)_{V} \times U(1)_{V}$ so that an identification with QCD can be made.

\section{A. The Pseudo-Goldstone Bosons}

The pseudo-Goldstone bosons of QQCD form a $6 \times 6$ matrix, $\Phi$, that can be written in block form

\footnotetext{
${ }^{1}$ A simple example of the implications of this distinction can be seen by considering the graded group $U(1 \mid 1)$ [15].
} 


$$
\Phi=\left(\begin{array}{cc}
\pi & \chi^{\dagger} \\
\chi & \tilde{\pi}
\end{array}\right)
$$

where $\pi$ is the $3 \times 3$ matrix of pseudo-Goldstone bosons including the $\eta^{\prime}$ with quantum numbers of $\bar{q} q$ pairs, $\tilde{\pi}$ is a $3 \times 3$ matrix of pseudo-Goldstone bosons including the $\tilde{\eta}^{\prime}$ with quantum numbers of $\overline{\tilde{q}} \tilde{q}$ pairs, and $\chi$ is a $3 \times 3$ matrix of pseudo-Goldstone fermions with quantum numbers of $\overline{\tilde{q}} q$ pairs,

$\pi=\left(\begin{array}{ccc}\eta_{u} & \pi^{+} & K^{+} \\ \pi^{-} & \eta_{d} & K^{0} \\ K^{-} & \bar{K}^{0} & \eta_{s}\end{array}\right) \quad, \quad \tilde{\pi}=\left(\begin{array}{ccc}\tilde{\eta}_{u} & \tilde{\pi}^{+} & \tilde{K}^{+} \\ \tilde{\pi}^{-} & \tilde{\eta}_{d} & \tilde{K}^{0} \\ \tilde{K}^{-} & \tilde{\tilde{K}}^{0} & \tilde{\eta}_{s}\end{array}\right) \quad, \quad \chi=\left(\begin{array}{ccc}\chi_{\eta_{u}} & \chi_{\pi^{+}} & \chi_{K^{+}} \\ \chi_{\pi^{-}} & \chi_{\eta_{d}} & \chi_{K^{0}} \\ \chi_{K^{-}} & \chi_{\bar{K}^{0}} & \chi_{\eta_{s}}\end{array}\right)$,

As the object

$$
\Phi_{0}=\frac{1}{\sqrt{6}} \operatorname{str}(\Phi)=\frac{1}{\sqrt{2}}\left(\eta^{\prime}-\tilde{\eta}^{\prime}\right)
$$

is invariant under $\left[S U(3 \mid 3)_{L} \otimes S U(3 \mid 3)_{R}\right] \times U(1)_{V}$ (but not under $\left.U(3 \mid 3)_{L} \otimes U(3 \mid 3)_{R}\right)$ the most general lagrange density that describes low-momentum dynamics will contain arbitrary functions of $\Phi_{0}$ [9, 10]. At lowest order in the chiral expansion, the Lagrange density that describes the dynamics of the pseudo-Goldstone bosons is, using the notation of Ref. [11],

$$
\mathcal{L}=\frac{f^{2}}{8} \operatorname{str}\left[\partial^{\mu} \Sigma^{\dagger} \partial_{\mu} \Sigma\right]+\lambda \operatorname{str}\left[m_{Q} \Sigma+m_{Q}^{\dagger} \Sigma^{\dagger}\right]+\alpha_{\Phi} \partial^{\mu} \Phi_{0} \partial_{\mu} \Phi_{0}-M_{0}^{2} \Phi_{0}^{2}
$$

where the parameter $\lambda$ is chosen to reproduce the meson masses, and $\Sigma$ is the exponential of the $\Phi$ field,

$$
\Sigma=\exp \left(\frac{2 i \Phi}{f}\right)
$$

With this normalization, $f \sim 132 \mathrm{MeV}$ in QCD. In addition, it is understood that the operators with coefficients $\alpha_{\Phi}$ and $M_{0}$, the hairpin interactions, are inserted perturbatively. Expanding out the Lagrange density in eq. (6) to quadratic order in the meson fields, one finds relations between the meson masses in the isospin limit,

$$
m_{\eta_{s}}^{2}=2 m_{K}^{2}-m_{\pi}^{2} \quad, \quad m_{\eta_{u}}^{2}=m_{\eta_{d}}^{2}=m_{\pi}^{2}
$$

The Lagrange density in eq. (6) has been used to compute several observables in the meson sector, such as $f_{K}, f_{\pi}$ [16] and the meson masses out to one-loop in perturbation theory [9, 10,17] (for a nice review see Ref. [18]).

\section{B. The Octet Baryons}

The inclusion of the lowest-lying baryons, the octet of spin- $\frac{1}{2}$ baryons and the decuplet of spin- $\frac{3}{2}$ baryon resonances, is detailed in Ref. [11]. An interpolating field that has non-zero overlap with the baryon octet (when the $i j k$ indices are restricted to $1,2,3$ ) is 11

$$
\mathcal{B}_{i j k}^{\gamma} \sim\left[Q_{i}^{\alpha, a} Q_{j}^{\beta, b} Q_{k}^{\gamma, c}-Q_{i}^{\alpha, a} Q_{j}^{\gamma, c} Q_{k}^{\beta, b}\right] \epsilon_{a b c}\left(C \gamma_{5}\right)_{\alpha \beta}
$$


where $C$ is the charge conjugation operator, $a, b, c$ are color indices and $\alpha, \beta, \gamma$ are Diracindices. Dropping the Dirac-index, one finds that under the interchange of flavor indices [11],

$$
\mathcal{B}_{i j k}=(-)^{1+\eta_{j} \eta_{k}} \mathcal{B}_{i k j}, \mathcal{B}_{i j k}+(-)^{1+\eta_{i} \eta_{j}} \mathcal{B}_{j i k}+(-)^{1+\eta_{i} \eta_{j}+\eta_{j} \eta_{k}+\eta_{k} \eta_{i}} \mathcal{B}_{k j i}=0 .
$$

In analogy with QCD, we consider the transformation of $B_{i j k}$ under $S U(3 \mid 3)_{V}$ transformations, and using the graded relation

$$
Q_{i} U_{k}^{j}=(-)^{\eta_{i}\left(\eta_{j}+\eta_{k}\right)} U_{k}^{j} Q_{i}
$$

in eq. (9), it is straightforward to show that [11

$$
\mathcal{B}_{i j k} \rightarrow(-)^{\eta_{l}\left(\eta_{j}+\eta_{m}\right)+\left(\eta_{l}+\eta_{m}\right)\left(\eta_{k}+\eta_{n}\right)} U_{i}^{l} U_{j}{ }^{m} U_{k}{ }^{n} \mathcal{B}_{l m n}
$$

The object $\mathcal{B}_{i j k}$ describes a $\mathbf{7 0}$ dimensional representation of $S U(3 \mid 3)_{V}$ [11]. It is convenient to decompose the irreducible representations of $S U(3 \mid 3)_{V}$ into irreducible representations $S U(3)_{q} \otimes S U(3)_{\tilde{q}} \otimes U(1)$ [19 21], and we will forget about the $U(1)$ 's from now on. The subscript denotes where the $S U(3)$ acts, either on the $q$ 's or on the $\tilde{q}$ 's. The ground floor of the 70-dimensional representation contains baryons that are comprised of three quarks, $q q q$, and is therefore an $(\mathbf{8}, \mathbf{1})$ of $S U(3)_{q} \otimes S U(3)_{\tilde{q}}$. It was shown in LS that the octet baryons are embedded as

$$
\mathcal{B}_{a b c}=\frac{1}{\sqrt{6}}\left(\epsilon_{a b d} B_{c}^{d}+\epsilon_{a c d} B_{b}^{d}\right)
$$

where the indices are restricted to take the values $a, b, c=1,2,3$ only. The octet baryon matrix is

$$
B=\left(\begin{array}{ccc}
\frac{1}{\sqrt{6}} \Lambda+\frac{1}{\sqrt{2}} \Sigma^{0} & \Sigma^{+} & p \\
\Sigma^{-} & \frac{1}{\sqrt{6}} \Lambda-\frac{1}{\sqrt{2}} \Sigma^{0} & n \\
\Xi^{-} & \Xi^{0} & -\frac{2}{\sqrt{6}} \Lambda
\end{array}\right)
$$

The first floor of the 70-dimensional representation contains baryons that are composed of two quarks and one ghost-quark, $\tilde{q} q q$, and therefore transforms as $(\mathbf{6}, \mathbf{3}) \oplus(\overline{\mathbf{3}}, \mathbf{3})$ of $S U(3)_{q} \otimes$ $S U(3)_{\tilde{q}}$. The tensor representation $\tilde{a} s_{a b}$ of the $(\mathbf{6}, \mathbf{3})$ multiplet, where $\tilde{a}=1,2,3$ runs over the $\tilde{q}$ indices and $a, b=1,2,3$ run over the $q$ indices, has baryon assignment

$$
\begin{aligned}
& \tilde{a}_{11}=\Sigma_{\tilde{a}}^{+1}, \quad s^{a} s_{12}={ }_{\tilde{a}} s_{21}=\frac{1}{\sqrt{2}} \Sigma_{\tilde{a}}^{0}, \quad \tilde{a}_{22}=\Sigma_{\tilde{a}}^{-1} \\
& \tilde{a} s_{13}={ }_{\tilde{a}} s_{31}=\frac{1}{\sqrt{2}}{ }^{(6)} \Xi_{\tilde{a}}^{+\frac{1}{2}}, \quad{ }_{a} s_{23}={ }_{\tilde{a}} s_{32}=\frac{1}{\sqrt{2}}{ }^{(6)} \Xi_{\tilde{a}}^{-\frac{1}{2}}, \quad \tilde{a}_{33}=\Omega_{\tilde{a}}^{0},
\end{aligned}
$$

where the notation closely follows that used to describe baryons containing a single heavy quark 2 . The right superscript denotes the third component of q-isospin, while the left

\footnotetext{
${ }^{2}$ Heavy baryons are classified by irreducible representations of $S U(2)_{H} \otimes S U(3)_{V}$, where $S U(2)_{H}$ is the $c, b$ heavy-quark-flavor symmetry group and $S U(3)_{V}$ is the light-quark flavor symmetry group.
} 
subscript denotes the $\tilde{q}$ flavor. The tensor representation ${ }_{a} t^{a}$ of the $(\overline{\mathbf{3}}, \mathbf{3})$ multiplet, where $\tilde{a}=1,2,3$ runs over the $\tilde{q}$ indices and $a=1,2,3$ run over the $q$ indices, has baryon assignment

$$
\tilde{a} t^{1}={ }^{(\overline{3})} \Xi_{\tilde{a}}^{-\frac{1}{2}}, \quad \tilde{a} t^{2}={ }^{(\overline{3})} \Xi_{\tilde{a}}^{+\frac{1}{2}}, \quad \tilde{a} t^{3}=\Lambda_{\tilde{a}}^{0}
$$

The $\tilde{a}_{a} s_{a b}$ and the $\tilde{a} t^{a}$ are uniquely embedded into $\mathcal{B}_{i j k}$ (up to field redefinitions), constrained by the relations in eq. (10):

$$
\begin{aligned}
& \mathcal{B}_{i j k}=\sqrt{\frac{2}{3}}{ }_{i-3} s_{j k} \quad \text { for } i=4,5,6 \text { and } j, k=1,2,3 \\
& \mathcal{B}_{i j k}=\frac{1}{2}{ }_{j-3} t^{\sigma} \varepsilon_{\sigma i k}+\frac{1}{\sqrt{6}}{ }_{j-3} s_{i k} \quad \text { for } \quad j=4,5,6 \text { and } i, k, \sigma=1,2,3 \\
& \mathcal{B}_{i j k}=-\frac{1}{2}{ }_{k-3} t^{\sigma} \varepsilon_{\sigma i j}-\frac{1}{\sqrt{6}}{ }_{k-3} s_{i j} \quad \text { for } \quad k=4,5,6 \text { and } i, j, \sigma=1,2,3 .
\end{aligned}
$$

As we are only interested in one-loop contributions to observables with $q q q$-baryons in the asymptotic states, we do not explicitly construct the second and third floors of the $\mathbf{7 0 .}$ However, it is straightforward to show that the second floor, consisting of $\tilde{q} \tilde{q} q$-baryons, can be decomposed into $(\mathbf{3}, \mathbf{6}) \oplus(\mathbf{3}, \overline{\mathbf{3}})$ of $S U(3)_{q} \otimes S U(3)_{\tilde{q}}$, while the third floor, consisting of $\tilde{q} \tilde{q} \tilde{q}$-baryons can be decomposed into a $(\mathbf{1}, \mathbf{8})$ of $S U(3)_{q} \otimes S U(3)_{\tilde{q}}$. To make contact with the discussions by LS [11], one can consider how the four floors transform under the vector $q+\tilde{q}$ symmetry of $S U(3)_{q} \otimes S U(3)_{\tilde{q}} \equiv S U(3)_{q+\tilde{q}} \otimes S U(3)_{q-\tilde{q}}$. The ground floor transforms as an $\mathbf{8}$ of $S U(3)_{q+\tilde{q}}$, the first floor transforms as an $(\mathbf{3} \otimes \mathbf{6}) \oplus(\mathbf{3} \otimes \overline{\mathbf{3}})=\mathbf{1 0} \oplus \mathbf{8} \oplus \mathbf{8} \oplus \mathbf{1}$, similarly the second floor transforms as an $\mathbf{1 0} \oplus \mathbf{8} \oplus \mathbf{8} \oplus \mathbf{1}$, and the third floor transforms as an $\mathbf{8}$.

\section{The Decuplet Baryons}

As the mass splitting between the decuplet and octet baryons (in QCD) is much less than the scale of chiral symmetry breaking $\left(\Lambda_{\chi} \sim 1 \mathrm{GeV}\right)$ the decuplet must be included as a dynamical field in order to have a theory where the natural scale of higher order interactions is set by $\Lambda_{\chi}$. We assume that the decuplet-octet mass splitting is still small compared to the scale of chiral symmetry breaking in QQCD. An interpolating field that contains the spin- $\frac{3}{2}$ decuplet as the ground floor is [11]

$$
\mathcal{T}_{i j k}^{\alpha, \mu} \sim\left[Q_{i}^{\alpha, a} Q_{j}^{\beta, b} Q_{k}^{\gamma, c}+Q_{i}^{\beta, b} Q_{j}^{\gamma, c} Q_{k}^{\alpha, a}+Q_{i}^{\gamma, c} Q_{j}^{\alpha, a} Q_{k}^{\beta, b}\right] \varepsilon_{a b c}\left(C \gamma^{\mu}\right)_{\beta \gamma} \quad,
$$

where the indices $i, j, k$ run from 1 to 6 . Neglecting Dirac-indices, one finds that under the interchange of flavor indices [1]]

$$
\mathcal{T}_{i j k}=(-)^{1+\eta_{i} \eta_{j}} \mathcal{T}_{j i k}=(-)^{1+\eta_{j} \eta_{k}} \mathcal{T}_{i k j}
$$

$\mathcal{T}_{i j k}$ describes a 38 dimensional representation of $S U(3 \mid 3)_{V}$, which has a ground floor transforming as $(\mathbf{1 0}, \mathbf{1})$ under $S U(3)_{q} \otimes S U(3)_{\tilde{q}}$ with

$$
\mathcal{T}_{a b c}=T_{a b c},
$$

where the indices are restricted to take the values $a, b, c=1,2,3$, and where $T_{a b c}$ is the totally symmetric tensor containing the decuplet of baryon resonances, 


$$
\begin{aligned}
& T_{111}=\Delta^{++}, T_{112}=\frac{1}{\sqrt{3}} \Delta^{+}, T_{122}=\frac{1}{\sqrt{3}} \Delta^{0}, T_{222}=\Delta^{-} \\
& T_{113}=\frac{1}{\sqrt{3}} \Sigma^{*,+}, T_{123}=\frac{1}{\sqrt{6}} \Sigma^{*, 0}, T_{223}=\frac{1}{\sqrt{3}} \Sigma^{*,-} \\
& T_{133}=\frac{1}{\sqrt{3}} \Xi^{*, 0}, T_{233}=\frac{1}{\sqrt{3}} \Xi^{*,-}, T_{333}=\Omega^{-} .
\end{aligned}
$$

The first floor transforms as a $(\mathbf{6}, \mathbf{3})$ under $S U(3)_{q} \otimes S U(3)_{\tilde{q}}$ which has a tensor representation, $\tilde{a} x_{i j}$, with baryon assignment

$$
\begin{aligned}
& \tilde{a} x_{11}=\Sigma_{\tilde{a}}^{*,+1}, \quad x_{a} x_{12}={ }_{\tilde{a}} x_{21}=\frac{1}{\sqrt{2}} \Sigma_{\tilde{a}}^{*, 0}, \quad x_{a} x_{22}=\Sigma_{\tilde{a}}^{*,-1} \\
& \tilde{a} x_{13}={ }_{\tilde{a}} x_{31}=\frac{1}{\sqrt{2}} \Xi_{\tilde{a}}^{*,+\frac{1}{2}}, \quad \tilde{a}_{23}={ }_{\tilde{a}} x_{32}=\frac{1}{\sqrt{2}} \Xi_{\tilde{a}}^{*,-\frac{1}{2}}, \quad{ }_{a} x_{33}=\Omega_{\tilde{a}}^{*, 0} .
\end{aligned}
$$

The embedding of ${ }_{a} x_{i j}$ into $\mathcal{T}_{i j k}$ is unique (up to field redefinitions), constrained by the symmetry properties in eq. (19):

$$
\begin{aligned}
& \mathcal{T}_{i j k}=+\frac{1}{\sqrt{3}}{ }_{i-3} x_{j k} \quad \text { for } \quad i=4,5,6 \text { and } j, k=1,2,3 \\
& \mathcal{T}_{i j k}=-\frac{1}{\sqrt{3}}{ }_{j-3} x_{i k} \quad \text { for } \quad j=4,5,6 \text { and } i, k=1,2,3 \\
& \mathcal{T}_{i j k}=+\frac{1}{\sqrt{3}}{ }_{k-3} x_{i j} \quad \text { for } \quad k=4,5,6 \text { and } i, j=1,2,3 \text {. }
\end{aligned}
$$

Again, we do not explicitly construct the second and third floor baryons of the 38 . However, it is easy to show that the second floor transforms as a $(\mathbf{3}, \overline{\mathbf{3}})$ and that the third floor transforms as a $(\mathbf{1}, \mathbf{1})$ under $S U(3)_{q} \otimes S U(3)_{\tilde{q}}$. Considering the transformation of the floors under $S U(3)_{q+\tilde{q}}$ we recover the results of LS, where the ground floor transforms as a $\mathbf{1 0}$, the first floor transforms as a $\mathbf{1 0} \oplus \mathbf{8}$, the second floor transforms as a $\mathbf{8} \oplus \mathbf{1}$ and the third floor transforms as a $\mathbf{1}$.

\section{Lagrange Density for the Baryons}

The free Lagrange density for the $\mathcal{B}_{i j k}$ and $\mathcal{T}_{i j k}$ fields is, at leading order in the heavy baryon expansion [22 26], and using the notation of LS [11],

$$
\begin{aligned}
\mathcal{L} & =i(\overline{\mathcal{B}} v \cdot \mathcal{D B})+2 \alpha_{M}\left(\overline{\mathcal{B}} \mathcal{B} \mathcal{M}_{+}\right)+2 \beta_{M}\left(\overline{\mathcal{B}} \mathcal{M}_{+} \mathcal{B}\right)+2 \sigma_{M}(\overline{\mathcal{B}} \mathcal{B}) \operatorname{str}\left(\mathcal{M}_{+}\right) \\
& -i\left(\overline{\mathcal{T}}^{\mu} v \cdot \mathcal{D} \mathcal{T}_{\mu}\right)+\Delta\left(\overline{\mathcal{T}}^{\mu} \mathcal{T}_{\mu}\right)+2 \gamma_{M}\left(\overline{\mathcal{T}}^{\mu} \mathcal{M}_{+} \mathcal{T}_{\mu}\right)-2 \bar{\sigma}_{M}\left(\overline{\mathcal{T}}^{\mu} \mathcal{T}_{\mu}\right) \operatorname{str}\left(\mathcal{M}_{+}\right)
\end{aligned}
$$

where $\mathcal{M}_{+}=\frac{1}{2}\left(\xi^{\dagger} m_{Q} \xi^{\dagger}+\xi m_{Q} \xi\right)$ (which differs by a factor of 2 from LS), $\Delta$ is the decupletoctet mass splitting, and $\xi=\sqrt{\Sigma}$. The brackets, ( ) denote contraction of lorentz and flavor indices as defined in LS [11]. For a matrix $\Gamma_{\beta}^{\alpha}$ acting in spin-space, and a matrix $Y_{i j}$ acting

in flavor-space (with or without lorentz indices), the required contractions are [11] 


$$
\begin{aligned}
(\overline{\mathcal{B}} \Gamma \mathcal{B}) & =\overline{\mathcal{B}}^{\alpha, k j i} \Gamma_{\alpha}^{\beta} \mathcal{B}_{i j k, \beta}, \quad\left(\overline{\mathcal{T}}^{\mu} \Gamma \mathcal{T}_{\mu}\right)=\overline{\mathcal{T}}^{\mu \alpha, k j i} \Gamma_{\alpha}^{\beta} \mathcal{T}_{i j k, \beta \mu} \\
(\overline{\mathcal{B}} \Gamma Y \mathcal{B}) & =\overline{\mathcal{B}}^{\alpha, k j i} \Gamma_{\alpha}^{\beta} Y_{i}{ }^{l} \mathcal{B}_{l j k, \beta}, \quad\left(\overline{\mathcal{T}}^{\mu} \Gamma Y \mathcal{T}_{\mu}\right)=\overline{\mathcal{T}}^{\mu \alpha, k j i} \Gamma_{\alpha}^{\beta} Y_{i}{ }^{l} \mathcal{T}_{l j k, \beta \mu} \\
(\overline{\mathcal{B}} \Gamma \mathcal{B} Y) & =(-)^{\left(\eta_{i}+\eta_{j}\right)\left(\eta_{k}+\eta_{n}\right)} \overline{\mathcal{B}}^{\alpha, k j i} \Gamma_{\alpha}^{\beta} Y_{k}{ }^{n} \mathcal{B}_{i j n, \beta} \\
\left(\overline{\mathcal{B}} \Gamma Y^{\mu} \mathcal{T}_{\mu}\right) & =\overline{\mathcal{B}}^{\alpha, k j i} \Gamma_{\alpha}^{\beta}\left(Y^{\mu}\right)_{i}^{l} \mathcal{T}_{l j k, \beta \mu},
\end{aligned}
$$

where $\overline{\mathcal{B}}$ and $\overline{\mathcal{T}}$ transform the same way,

$$
\bar{B}^{k j i} \rightarrow(-)^{\eta_{l}\left(\eta_{j}+\eta_{m}\right)+\left(\eta_{l}+\eta_{m}\right)\left(\eta_{k}+\eta_{n}\right)} \overline{\mathcal{B}}^{n m l} U_{n}{ }^{k \dagger} U_{m}^{j \dagger} U_{l}^{i \dagger} .
$$

The Lagrange density describing the interactions of the baryons with the pseudoGoldstone bosons is [11]

$$
\begin{aligned}
& \mathcal{L}=2 \alpha\left(\overline{\mathcal{B}} S^{\mu} \mathcal{B} A_{\mu}\right)+2 \beta\left(\overline{\mathcal{B}} S^{\mu} A_{\mu} \mathcal{B}\right)+2 \gamma\left(\overline{\mathcal{B}} S^{\mu} \mathcal{B}\right) \operatorname{str}\left(A_{\mu}\right) \\
& +2 \mathcal{H}\left(\overline{\mathcal{T}}^{\nu} S^{\mu} A_{\mu} \mathcal{T}_{\nu}\right)+\sqrt{\frac{3}{2}} \mathcal{C}\left[\left(\overline{\mathcal{T}}^{\nu} A_{\nu} \mathcal{B}\right)+\left(\mathcal{B} A_{\nu} \mathcal{T}^{\nu}\right)\right]+2 \gamma^{\prime}\left(\overline{\mathcal{T}}^{\nu} S^{\mu} \mathcal{T}_{\nu}\right) \operatorname{str}\left(A_{\mu}\right)
\end{aligned}
$$

where $S^{\mu}$ is the covariant spin-vector [22 24], and where the sign of the $\mathcal{C}$-term is opposite to that of LS P. Restricting oneself to the $q q q$ sector, it is straightforward to show that

$$
\alpha=\frac{2}{3} D+2 F \quad, \quad \beta=-\frac{5}{3} D+F,
$$

where $D$ and $F$ are constants that multiply the $S U(3)_{q}$ invariants that are commonly used in QCD, and it should be stressed that the $F$ and $D$ discussed here will not have the numerical values of those of QCD. In QQCD there is an additional coupling, $\gamma$, a hairpin interaction [11] that is not usually considered in the $\chi \mathrm{PT}$ description of low-energy QCD. In our calculation of the magnetic moments we will replace $\alpha$ and $\beta$ with $F$ and $D$, but we will keep $\gamma$ explicit, as in LS [11]. In the above discussion, vector and axial-vector meson fields have been introduced in direct analogy with QCD. The covariant derivative acting on either the $\mathcal{B}$ or $\mathcal{T}$ fields has the form

$$
\left(\mathcal{D}^{\mu} \mathcal{B}\right)_{i j k}=\partial^{\mu} \mathcal{B}_{i j k}+\left(V^{\mu}\right)_{i}^{l} \mathcal{B}_{l j k}+(-)^{\eta_{i}\left(\eta_{j}+\eta_{m}\right)}\left(V^{\mu}\right)_{j}^{m} \mathcal{B}_{i m k}+(-)^{\left(\eta_{i}+\eta_{j}\right)\left(\eta_{k}+\eta_{n}\right)}\left(V^{\mu}\right)_{k}^{n} \mathcal{B}_{i j n}
$$

where the vector and axial-vector meson fields are

$$
V^{\mu}=\frac{1}{2}\left(\xi \partial^{\mu} \xi^{\dagger}+\xi^{\dagger} \partial^{\mu} \xi\right), A^{\mu}=\frac{i}{2}\left(\xi \partial^{\mu} \xi^{\dagger}-\xi^{\dagger} \partial^{\mu} \xi\right)
$$

It is useful to expand the interactions of the ground and first floors,

\footnotetext{
3 The sign of $\mathcal{C}$ has no physical meaning as a field redefinition on either baryon field, $\mathcal{B} \rightarrow-\mathcal{B}$ or $\mathcal{T} \rightarrow-\mathcal{T}$ changes the sign of the $\mathcal{C}$-term.
} 


$$
\begin{aligned}
\left(\overline{\mathcal{B}} S^{\mu} \mathcal{B} A_{\mu}\right)= & \frac{1}{f}\left[\frac{1}{6} \operatorname{Tr}\left[\bar{B} S^{\mu} B\right] \operatorname{Tr}\left[\partial_{\mu} \pi\right]-\frac{1}{6} \operatorname{Tr}\left[\bar{B} S^{\mu} B \partial_{\mu} \pi\right]+\frac{2}{3} \operatorname{Tr}\left[\bar{B} S^{\mu} \partial_{\mu} \pi B\right]\right. \\
& +\frac{1}{6} \epsilon^{n i j} \bar{B}_{n}^{k} S^{\mu} \partial_{\mu} \chi_{i}^{\tilde{a} \dagger} \tilde{a}_{j} s_{j k}+\frac{1}{6} \epsilon_{n i j}{ }^{\tilde{a}} \bar{s}^{j k} S^{\mu} \partial_{\mu} \chi_{\tilde{a}}^{i} B_{k}^{n} \\
& \left.-\sqrt{\frac{3}{8}} \operatorname{Tr}\left[\bar{B} S^{\mu} \partial_{\mu} \chi^{\dagger} t\right]-\sqrt{\frac{3}{8}} \operatorname{Tr}\left[\bar{t} S^{\mu} \partial_{\mu} \chi B\right]\right]+\ldots \\
\left(\overline{\mathcal{B}} S^{\mu} A_{\mu} \mathcal{B}\right)= & \frac{1}{f}\left[\frac{2}{3} \operatorname{Tr}\left[\bar{B} S^{\mu} B\right] \operatorname{Tr}\left[\partial_{\mu} \pi\right]-\frac{2}{3} \operatorname{Tr}\left[\bar{B} S^{\mu} B \partial_{\mu} \pi\right]-\frac{1}{3} \operatorname{Tr}\left[\bar{B} S^{\mu} \partial_{\mu} \pi B\right]\right. \\
& \left.+\frac{2}{3} \epsilon^{n i j} \bar{B}_{n}^{k} S^{\mu} \partial_{\mu} \chi_{i}^{\tilde{a} \dagger} \tilde{a}_{\tilde{a}} s_{j k}+\frac{2}{3} \epsilon_{n i j}{ }^{\tilde{a}} \bar{s}^{j k} S^{\mu} \partial_{\mu} \chi_{\tilde{a}}^{i} B_{k}^{n}\right]+\ldots \\
\left(\overline{\mathcal{B}} S^{\mu} \mathcal{B}\right) \operatorname{str}\left(A_{\mu}\right)= & \frac{1}{f}\left[\operatorname{Tr}\left[\bar{B} S^{\mu} B\right]+\operatorname{Tr}\left[\bar{t} S^{\mu} t\right]+\operatorname{Tr}\left[\bar{s} S^{\mu} s\right]\right] \operatorname{Tr}\left[\partial_{\mu} \pi-\partial \tilde{\pi}\right]+\ldots \\
\left(\overline{\mathcal{B}} A^{\mu} \mathcal{T}_{\mu}\right)= & \frac{1}{f} \sqrt{\frac{2}{3}} \epsilon^{x i j}\left[\bar{B}_{x}^{k} \partial^{\mu} \pi_{i}^{l} T_{l j k}+\frac{1}{\sqrt{3}} \bar{B}_{x}^{k} \partial^{\mu} \chi_{i}^{\tilde{a} \dagger} x_{j k}\right]+\ldots
\end{aligned}
$$

where all the indices that appear in eq. (31) are restricted to be 1,2,3. Terms that do not contribute at one-loop level to the magnetic moments of the octet baryons have not been shown in eq. (31).

The appropriate one-loop diagrams resulting from the Lagrange density in eqs. (6), (24) and (27) reproduce both the $\mathcal{O}\left(m_{q}^{3 / 2}\right)$, the $\mathcal{O}\left(\alpha_{\Phi} m_{q}^{3 / 2}\right)$ and the $\mathcal{O}\left(M_{0}^{2} m_{q}^{1 / 2}\right)$ contributions to the masses of the octet baryons (the last two coming from hairpin diagrams) as calculated in LS [11] using quark-line diagrams. We have not computed the higher order contributions to the octet baryon masses, nor the contributions to the decuplet masses, but expect that we would recover the results of LS [11].

\section{MAGNETIC MOMENTS OF THE OCTET BARYONS}

The magnetic moments of the octet baryons have been studied extensively in QCD. Coleman and Glashow (CG) [27] first considered the moments in the limit of exact $S U(3)$ flavor symmetry, arriving at six relations between the moments and the $\Sigma^{0}-\Lambda$ transition moment, known as the Coleman-Glashow relations. The leading dependence upon the light quark masses, of the form $\sqrt{m_{q}}$, was first computed by Caldi and Pagels [3], and more recently higher order contributions have been computed including the decuplet as a dynamical degree of freedom [⿴囗十 $\left[7\right.$. In QQCD one expects that $\sim \sqrt{m_{q}}$ terms are suppressed as only quarks in the asymptotic states can participate in meson loops. However, hairpin interactions, and in particular those arising from the operator with coefficient $M_{0}^{2}$ in eq. (6), give rise to contributions of the form $\sim M_{0}^{2} \log m_{q}$, due to the more singular behavior of QQCD in the chiral limit than QCD.

\section{A. Tree-Level}

The leading order contribution to the magnetic moments of the octet baryons arises from two dimension-5 operators, 


$$
\begin{aligned}
\mathcal{L} & =\frac{e}{4 M_{N}} F_{\mu \nu}\left[\mu_{\alpha}\left(\overline{\mathcal{B}} \sigma^{\mu \nu} \mathcal{B} \mathcal{Q}_{\xi+}\right)+\mu_{\beta}\left(\overline{\mathcal{B}} \sigma^{\mu \nu} \mathcal{Q}_{\xi+} \mathcal{B}\right)\right] \\
\mathcal{Q}_{\xi+} & =\frac{1}{2}\left(\xi^{\dagger} \mathcal{Q} \xi+\xi \mathcal{Q} \xi^{\dagger}\right)
\end{aligned}
$$

where $\mathcal{Q}$ is the electric charge matrix, $\mathcal{Q}=\frac{1}{3} \operatorname{diag}(+2,-1,-1,+2,-1,-1)$. By considering only the ground floor baryons one can make the identification with the flavor structure of operators used in QCD

$$
\mathcal{L}=\frac{e}{4 M_{N}} F_{\mu \nu}\left(\mu_{D} \operatorname{Tr}\left[\bar{B} \sigma^{\mu \nu}\{Q, B\}\right]+\mu_{F} \operatorname{Tr}\left[\bar{B} \sigma^{\mu \nu}[Q, B]\right]\right)+\ldots,
$$

where the ellipses denotes terms involving the meson field, and find that

$$
\mu_{\alpha}=\frac{2}{3} \mu_{D}+2 \mu_{F} \quad, \quad \mu_{\beta}=-\frac{5}{3} \mu_{D}+\mu_{F} .
$$

Again, one should keep in mind that the values of $\mu_{D}$ and $\mu_{F}$ in QQCD will differ from those in QCD. The Lagrange density in eq. (32) not only generates magnetic moments for the octet baryons but also for the entire $\mathbf{7 0}$ dimensional representation. In Table $\llbracket$ we show

\begin{tabular}{cc}
\hline \hline Baryon & $\mu_{i}^{\text {tree }}(\mathrm{NM})$ \\
\hline$p, \Sigma^{+}$ & $\frac{1}{3} \mu_{D}+\mu_{F}$ \\
$n, \Xi^{0}$ & $-\frac{2}{3} \mu_{D}$ \\
$\Sigma^{0}$ & $\frac{1}{3} \mu_{D}$ \\
$\Sigma^{-}, \Xi^{-}$ & $\frac{1}{3} \mu_{D}-\mu_{F}$ \\
$\Lambda$ & $-\frac{1}{3} \mu_{D}$ \\
$\Sigma^{0}-\Lambda$-transition & $\frac{1}{\sqrt{3}} \mu_{D}$ \\
\hline \hline
\end{tabular}

TABLE I. The tree-level contribution to the magnetic moments of the octet baryons and the $\Sigma^{0}-\Lambda$ transition moment (i.e. the ground floor of the $\mathbf{7 0}$ ) in units of nuclear magnetons (NM).

the tree-level magnetic moments of the octet baryons and $\Sigma^{0}-\Lambda$ transition moment in units of nuclear magnetons. We have not shown the tree-level contributions to the first or higher floors of the $\mathbf{7 0}$ as they do not contribute to the magnetic moments of the octet baryons at one-loop.

As there are only two dimension-5 invariants in QQCD, the same number as in QCD, the CG relations [27 between the magnetic moments of the octet baryons in the limit of exact $S U(3)_{V}$ flavor symmetry persist:

$$
\begin{array}{ll}
\mu_{\Sigma^{+}}=\mu_{p} & , \quad \mu_{\Sigma^{-}}+\mu_{n}=-\mu_{p} \\
2 \mu_{\Lambda}=\mu_{n} & , \quad \mu_{\Xi^{-}}=\mu_{\Sigma^{-}} \\
\mu_{\Xi^{0}}=\mu_{n} & , \quad 2 \mu_{\Sigma^{0} \Lambda}=-\sqrt{3} \mu_{n} .
\end{array}
$$

\section{B. Contributions of the form $\sim \sqrt{m_{q}}$}

In QCD the leading contributions to the magnetic moments that depend upon the lightquark masses arise from the one-loop graphs shown in fig. [1. The photon couples to a 


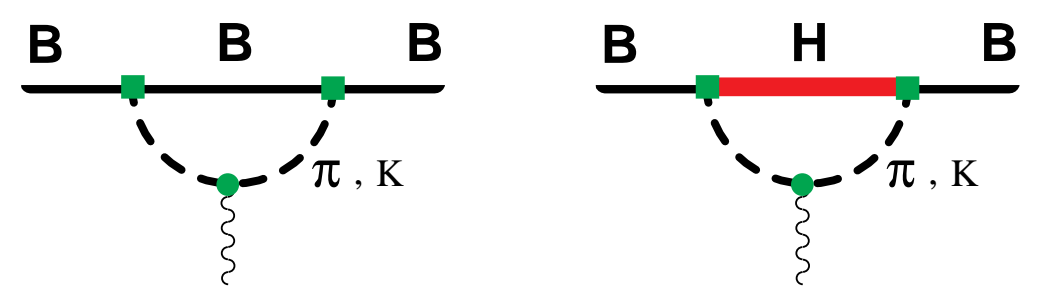

FIG. 1. One-loop graphs that give contributions of the form $\sim \sqrt{m_{q}}$ to the magnetic moments of the octet baryons in QCD. A solid, thick-solid and dashed line denote an octet baryon, decuplet baryon, and a meson, respectively. The wiggly line denotes a photon. The solid-squares, solid-circles denote an axial, minimal electromagnetic coupling, respectively. In QQCD, in addition to the intermediate states involving an octet or decuplet baryon and a charged pseudo-scalar meson, there are contributions from ghost-baryons, the t's, $s$ 's and $x$ 's, and fermionic pseudo-scalar mesons, the $\chi_{\pi^{ \pm}}$and $\chi_{K^{ \pm}}$.

charged meson which is coupled to the baryon via axial interactions. Graphs with octet baryons in the intermediate state give contributions of the form $\sim \sqrt{m_{q}}$, whereas those with decuplet intermediate states have a more complicated functional dependence upon $m_{q}$ due to the presence of the decuplet-octet mass splitting, $\Delta$. In QQCD the diagrams shown in fig. 1 also generate contributions of the form $\sim \sqrt{m_{q}}$, but in addition to intermediate states of the ground floor of baryons and pseudo-scalar mesons, $\pi^{ \pm}$and $K^{ \pm}$, there are contributions from intermediate states of ghost-baryons, the $t$ 's, $s$ 's and $x$ 's, and fermionic mesons, the $\chi_{\pi^{ \pm}}$and $\chi_{K^{ \pm}}$.

Following Ref. [4], we write the contribution to the magnetic moments from these diagrams as

$$
\delta \mu_{i}=\frac{M_{N}}{4 \pi f^{2}} \sum_{X=\pi, K}\left[\beta_{i}^{(X)} m_{X}+\beta_{i}^{(X)} \mathcal{F}\left(m_{X}, \Delta, \mu\right)\right]
$$

which is true in both QCD and QQCD (Ref. [4 uses $f \sim 94 \mathrm{MeV}$ and hence the coefficients in eq. (36) differ from those of Ref. 四 by a factor of 2). The $\beta_{i}^{(X)}$ contributions arise from diagrams with baryons in the $\mathbf{7 0}$ dimensional representation in the intermediate state, while the $\beta_{i}^{(X)}$ contributions arise from diagrams with baryons in the $\mathbf{3 8}$ dimensional representation in the intermediate state. The loop-function $\mathcal{F}\left(m_{X}, \Delta, \mu\right)$ is defined to be $\mathbb{\|}$

$$
\pi \mathcal{F}(m, \Delta, \mu)=\sqrt{\Delta^{2}-m^{2}} \log \left(\frac{\Delta-\sqrt{\Delta^{2}-m^{2}+i \epsilon}}{\Delta+\sqrt{\Delta^{2}-m^{2}+i \epsilon}}\right)-\Delta \log \left(\frac{m^{2}}{\mu^{2}}\right)
$$

where $\mu$ is the renormalization scale, and $\mathcal{F}(m, 0, \mu)=m$.

The computed values of the coefficients $\beta^{(\pi)}, \beta^{(K)}, \beta^{\prime(\pi)}$ and $\beta^{\prime(K)}$ that appear in eq. (36) are shown in Table [I and Table [II for both QCD and QQCD. It is interesting to note that, unlike QCD, there is no contribution from the F-type axial interaction in QQCD. In QCD, there are three relations between the moments of the octet baryons that hold in the presence of the $\sim \sqrt{m_{q}}$ contributions, as first pointed out by Caldi and Pagels (CP) [3] for octet baryons in the intermediate state, and subsequently shown to be valid for decuplet baryons in the intermediate state [4], 


\begin{tabular}{|c|c|c|c|c|}
\hline \multirow[b]{2}{*}{ Baryon } & \multicolumn{2}{|c|}{ QCD } & \multicolumn{2}{|c|}{ QQCD } \\
\hline & $\beta^{(\pi)}$ & $\beta^{(K)}$ & $\beta^{(\pi)}$ & $\beta^{(K)}$ \\
\hline$p$ & $-(D+F)^{2}$ & $-\frac{2}{3} D^{2}-2 F^{2}$ & $-\frac{4}{3} D^{2}$ & 0 \\
\hline$n$ & $(D+F)^{2}$ & $-(D-F)^{2}$ & $\frac{4}{3} D^{2}$ & 0 \\
\hline$\Sigma^{+}$ & $-\frac{2}{3} D^{2}-2 F^{2}$ & $-(D+F)^{2}$ & 0 & $-\frac{4}{3} D^{2}$ \\
\hline$\Sigma^{0}$ & 0 & $-2 D F$ & 0 & $-\frac{2}{3} D^{2}$ \\
\hline$\Sigma^{-}$ & $\frac{2}{3} D^{2}+2 F^{2}$ & $(D-F)^{2}$ & 0 & 0 \\
\hline$\Lambda$ & 0 & $2 D F$ & 0 & $\frac{2}{3} D^{2}$ \\
\hline$\Xi^{-}$ & $(D-F)^{2}$ & $\frac{2}{3} D^{2}+2 F^{2}$ & 0 & 0 \\
\hline$\Xi^{0}$ & $-(D-F)^{2}$ & $(D+F)^{2}$ & 0 & $\frac{4}{3} D^{2}$ \\
\hline$\Sigma^{0} \Lambda$ & $-\frac{4}{\sqrt{3}} D F$ & $-\frac{2}{\sqrt{3}} D F$ & $-\frac{4}{3 \sqrt{3}} D^{2}$ & $-\frac{2}{3 \sqrt{3}} D^{2}$ \\
\hline
\end{tabular}

TABLE II. The coefficients $\beta^{(\pi)}$ and $\beta^{(K)}$ in QCD and QQCD arising from the one-loop graphs shown in fig. 1 .

\begin{tabular}{ccccc}
\hline \hline & & QCD & & QQCD \\
Baryon & $\beta^{\prime(\pi)}$ & $\beta^{\prime(K)}$ & $\beta^{\prime(\pi)}$ & $\beta^{\prime(K)}$ \\
\hline$p$ & $-\frac{2}{9} \mathcal{C}^{2}$ & $\frac{1}{18} \mathcal{C}^{2}$ & $-\frac{1}{6} \mathcal{C}^{2}$ & 0 \\
$n$ & $\frac{2}{9} \mathcal{C}^{2}$ & $\frac{1}{9} \mathcal{C}^{2}$ & $\frac{1}{6} \mathcal{C}^{2}$ & $-\frac{1}{6} \mathcal{C}^{2}$ \\
$\Sigma^{+}$ & $\frac{1}{18} \mathcal{C}^{2}$ & $-\frac{2}{9} \mathcal{C}^{2}$ & 0 & $-\frac{1}{12} \mathcal{C}^{2}$ \\
$\Sigma^{0}$ & 0 & $-\frac{1}{6} \mathcal{C}^{2}$ & 0 & 0 \\
$\Sigma^{-}$ & $-\frac{1}{18} \mathcal{C}^{2}$ & $-\frac{1}{9} \mathcal{C}^{2}$ & 0 & $\frac{1}{12} \mathcal{C}^{2}$ \\
$\Lambda$ & 0 & $\frac{1}{6} \mathcal{C}^{2}$ & 0 & 0 \\
$\Xi^{-}$ & $-\frac{1}{9} \mathcal{C}^{2}$ & $-\frac{1}{18} \mathcal{C}^{2}$ & 0 & $\frac{1}{6} \mathcal{C}^{2}$ \\
$\Xi^{0}$ & $\frac{1}{9} \mathcal{C}^{2}$ & $\frac{2}{9} \mathcal{C}^{2}$ & 0 & 1 \\
$\Sigma^{0} \Lambda$ & $-\frac{1}{3 \sqrt{3}} \mathcal{C}^{2}$ & $-\frac{1}{6 \sqrt{3}} \mathcal{C}^{2}$ & $-\frac{1}{6 \sqrt{3}} \mathcal{C}^{2}$ & $-\frac{1}{12 \sqrt{3}} \mathcal{C}^{2}$ \\
\hline \hline
\end{tabular}

TABLE III. The coefficients $\beta^{\prime(\pi)}$ and $\beta^{\prime(K)}$ in QCD and QQCD arising from the one-loop graphs shown in fig. 1.

$$
\mu_{\Sigma^{+}}=-2 \mu_{\Lambda}-\mu_{\Sigma^{-}}, \quad \mu_{\Lambda}-\sqrt{3} \mu_{\Sigma^{0} \Lambda}=\mu_{\Xi^{0}}+\mu_{n} \quad, \quad \mu_{\Xi^{0}}+\mu_{\Xi^{-}}+\mu_{n}=2 \mu_{\Lambda}-\mu_{p},
$$

which are found to hold at the $\sim 10 \%$-level in nature [4]. This is to be compared with the CG-relations [27], that are found to hold at the $\sim 30 \%$-level, consistent with naive expectations. The CP-relations are also true in QQCD when only the $\sim \sqrt{m_{q}}$ contributions are considered, as can be seen with ease in Table II and Table III. It is not surprising to see that the size of the loop corrections in QQCD are smaller than their counterparts in QCD. After all, quenching removes quark loop contributions. A consequence of this is that the loop expansion for the magnetic moments is expected to be more convergent, and hopefully valid for higher quark masses in QQCD than in QCD, as it should be for all observables.

\section{Contributions of the form $M_{0}^{2} \log m_{q}$ : Hairpins}

Unlike QCD, in QQCD there are contributions to the magnetic moments of the baryons of the form $\sim M_{0}^{2} \log m_{q}$ from the diagrams in fig. 2, where $M_{0}$ is one of the hairpin interactions 
defined in eq. (6). Of course, there are other hairpin interactions that will contribute, but this one gives the terms that are most singular in the chiral limit. For these hairpin contributions,
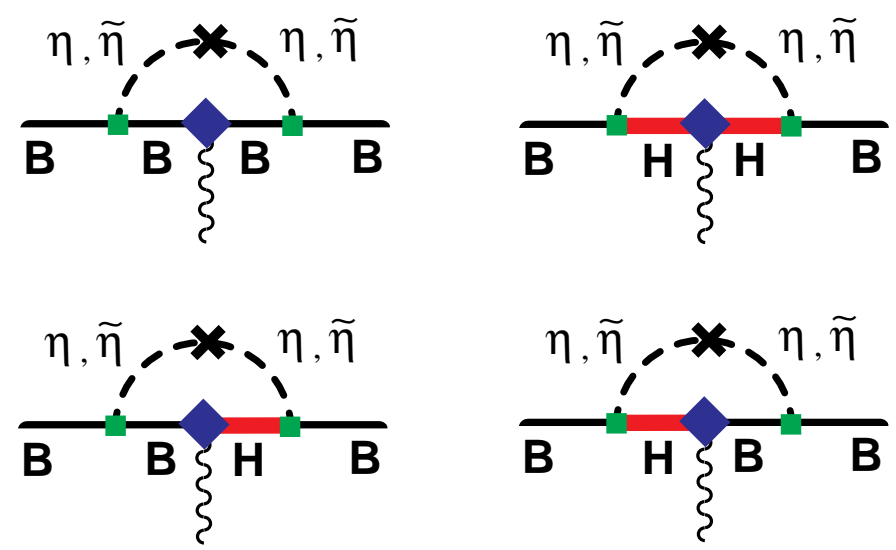

(a)
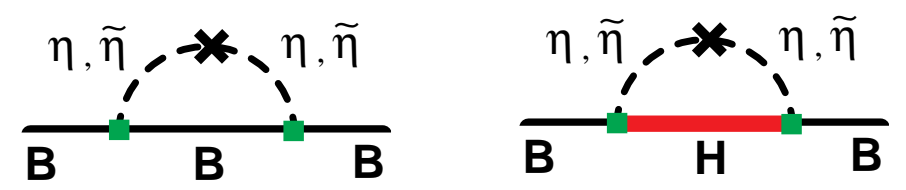

(b)

FIG. 2. The one-loop graphs that give contributions of the form $\sim M_{0}^{2} \log m_{q}$ to the magnetic moments of the octet baryons in QQCD. A solid, thick-solid, dashed line denotes an octet baryon, decuplet baryon, meson, respectively. A wiggly line denotes a photon. A cross on a meson line corresponds to an insertion of the hairpin interaction with coefficient $M_{0}$, while the solid-diamonds, solid-squares correspond to magnetic moment, axial interactions, respectively. Diagrams (a) are vertex corrections while diagrams (b) give rise to wavefunction renormalization.

only the ground floor baryons contribute, but both $\eta_{q}$ and $\tilde{\eta}_{q}$ mesons can appear in the loop. As discussed in Ref. [28], the contributions from the axial hairpin interaction, with coefficient $\gamma$, in such diagrams vanish, leaving only contributions from the $F, D$ and $\mathcal{C}$ interactions.

The wavefunction renormalization factors arising from the diagrams shown in fig. 2(b) are

$$
\begin{aligned}
& Z_{\psi}^{N}=1-\frac{m_{0}^{2}}{16 \pi^{2} f^{2}}(D-3 F)^{2} \bar{I}_{u u} \\
& Z_{\psi}^{\Sigma}=1-\frac{m_{0}^{2}}{16 \pi^{2} f^{2}}\left[4 F^{2} \bar{I}_{u u}+(D-F)^{2} \bar{I}_{s s}+4 F(F-D) \bar{I}_{u s}+\frac{2 \mathcal{C}^{2}}{9}\left(\bar{I}_{s s}^{\Delta \Delta}+\bar{I}_{u u}^{\Delta \Delta}-2 \bar{I}_{u s}^{\Delta \Delta}\right)\right] \\
& Z_{\psi}^{\Xi}=1-\frac{m_{0}^{2}}{16 \pi^{2} f^{2}}\left[4 F^{2} \bar{I}_{s s}+(D-F)^{2} \bar{I}_{u u}+4 F(F-D) \bar{I}_{u s}+\frac{2 \mathcal{C}^{2}}{9}\left(\bar{I}_{s s}^{\Delta \Delta}+\bar{I}_{u u}^{\Delta \Delta}-2 \bar{I}_{u s}^{\Delta \Delta}\right)\right] \\
& Z_{\psi}^{\Lambda}=1-\frac{m_{0}^{2}}{16 \pi^{2} f^{2}}\left[\frac{4}{9}(2 D-3 F)^{2} \bar{I}_{u u}-\frac{4}{9}\left(2 D^{2}+3 D F-9 F^{2}\right) \bar{I}_{u s}+\frac{1}{9}(D+3 F)^{2} \bar{I}_{s s}\right],
\end{aligned}
$$


where the contribution to an S-matrix element is $\sim \sqrt{Z_{\psi}^{i}} \sqrt{Z_{\psi}^{f}} \Gamma^{\text {tree }}$, where $\Gamma^{\text {tree }}$ is the treelevel vertex and $i, f$ denote the initial and final state baryon, respectively. The integrals that appear in eq. (39) are defined to be

$$
\bar{I}_{q q^{\prime}}=\bar{I}\left(m_{\eta_{q}}, m_{\eta_{q^{\prime}}}, 0,0, \mu\right), \bar{I}_{q q^{\prime}}^{\Delta}=\bar{I}\left(m_{\eta_{q}}, m_{\eta_{q^{\prime}}}, \Delta, 0, \mu\right), \bar{I}_{q q^{\prime}}^{\Delta \Delta}=\bar{I}\left(m_{\eta_{q}}, m_{\eta_{q^{\prime}}}, \Delta, \Delta, \mu\right)
$$

where

$$
\begin{gathered}
\bar{I}\left(m_{1}, m_{2}, \Delta_{1}, \Delta_{2}, \mu\right)=\frac{\left[Y\left(m_{1}, \Delta_{1}, \mu\right)+Y\left(m_{2}, \Delta_{2}, \mu\right)-Y\left(m_{1}, \Delta_{2}, \mu\right)-Y\left(m_{2}, \Delta_{1}, \mu\right)\right]}{\left[\Delta_{1}-\Delta_{2}\right]\left[m_{1}^{2}-m_{2}^{2}\right]} \\
=-i \frac{16 \pi^{2}}{3} \int \frac{d^{n} q}{(2 \pi)^{n}} \frac{q^{2}-(v \cdot q)^{2}}{\left[v \cdot q-\Delta_{1}+i \epsilon\right]\left[v \cdot q-\Delta_{2}+i \epsilon\right]\left[q^{2}-m_{1}^{2}+i \epsilon\right]\left[q^{2}-m_{2}^{2}+i \epsilon\right]},
\end{gathered}
$$

with

$Y(m, \Delta, \mu)=\left[m^{2}-\frac{2}{3} \Delta^{2}\right] \Delta \log \left(\frac{m^{2}}{\mu^{2}}\right)+\frac{2}{3}\left[\Delta^{2}-m^{2}\right]^{\frac{3}{2}} \log \left(\frac{\Delta-\sqrt{\Delta^{2}-m^{2}+i \epsilon}}{\Delta+\sqrt{\Delta^{2}-m^{2}+i \epsilon}}\right)$.

The integral in eq. (41) has nice limits, e.g.

$$
\begin{aligned}
\bar{I}\left(m_{1}, m_{2}, 0,0, \mu\right) & =\frac{m_{1}^{2} \log \left(\frac{m_{1}^{2}}{\mu^{2}}\right)-m_{2}^{2} \log \left(\frac{m_{2}^{2}}{\mu^{2}}\right)}{m_{1}^{2}-m_{2}^{2}}+\ldots \\
\bar{I}(m, m, 0,0, \mu) & =\log \left(\frac{m^{2}}{\mu^{2}}\right)+\ldots
\end{aligned}
$$

where the ellipses denote terms that are analytic in $m_{q}$. In computing the loop graphs we have performed the spin algebra in four dimensions.

In the diagrams shown in fig. 2 one sees that there are contributions from the magnetic moments of the decuplet baryons, and from the transition magnetic moment between the decuplet and octet baryons. The Lagrange density that describes these interactions is [4]

$$
\begin{aligned}
\mathcal{L}= & -i \sqrt{\frac{3}{2}} \mu_{T} \frac{e}{2 M_{N}} F_{\mu \nu}\left[\left(\overline{\mathcal{B}} S^{\mu} \mathcal{Q}_{\xi+} \mathcal{T}^{\nu}\right)+\text { h.c. }\right]-i 3 \mu_{C} \frac{e}{2 M_{N}} F_{\mu \nu}\left(\overline{\mathcal{T}}^{\mu} \mathcal{Q}_{\xi+} \mathcal{T}^{\nu}\right) \\
= & i \mu_{T} \frac{e}{2 M_{N}} F_{\mu \nu}\left(\epsilon_{i j k} Q_{l}^{i} \bar{B}_{m}^{j} S^{\mu} T^{\nu, k l m}+\epsilon^{i j k} Q_{i}^{l} \bar{T}_{k l m}^{\mu} S^{\nu} B_{j}^{m}\right)-i \mu_{C} \frac{e}{M_{N}} q_{i} \bar{T}_{i}^{\mu} T_{i}^{\nu} F_{\mu \nu} \\
& +\ldots
\end{aligned}
$$

where $q_{i}$ is the charge of the $i^{\text {th }}$ decuplet baryon, and the ellipses denote terms involving meson fields. The expressions that one obtains for the hairpin contribution to the magnetic moment of each baryon can be written as

$\delta \mu_{i}=\frac{M_{0}^{2}}{16 \pi^{2} f^{2}}\left[-\frac{4}{3} \mu_{i}^{\text {tree }} w_{i}^{(8,8)}+\mathcal{C} \mu_{T} w_{i}^{(8,10)}+\mathcal{C}^{2}\left(\bar{I}_{s s}^{\Delta \Delta}+\bar{I}_{u u}^{\Delta \Delta}-2 \bar{I}_{u s}^{\Delta \Delta}\right) w_{i}^{(10,10)}\right]$,

where $w_{i}^{(8,8)}$ is the contribution from intermediate states with octet baryons, $w_{i}^{(10,10)}$ is the contribution from intermediate states with decuplet baryons, and $w_{i}^{(8,10)}$ is the contribution from intermediate states with both octet and decuplet baryons. The expressions that we 


\begin{tabular}{cc}
\hline \hline Baryon & $w_{i}^{(8,8)}$ \\
\hline$p, n$ & $(D-3 F)^{2} \bar{I}_{u u}$ \\
$\Sigma^{+}, \Sigma^{0}, \Sigma^{-}$ & $4 F^{2} \bar{I}_{u u}+(D-F)^{2} \bar{I}_{s s}+4 F(F-D) \bar{I}_{u s}$ \\
$\Lambda$ & $\frac{1}{9}\left(4(2 D-3 F)^{2} \bar{I}_{u u}-4\left(2 D^{2}+3 D F-9 F^{2}\right) \bar{I}_{u s}+(D+3 F)^{2} \bar{I}_{s s}\right)$ \\
$\Xi^{0}, \Xi^{-}$ & $4 F^{2} \bar{I}_{s s}+(D-F)^{2} \bar{I}_{u u}+4 F(F-D) \bar{I}_{u s}$ \\
$\Sigma^{0} \rightarrow \Lambda$ & $\left(4 F^{2}-\frac{8}{3} D F+\frac{2}{3} D^{2}\right) \bar{I}_{u u}+\left(F^{2}-\frac{2}{3} D F+\frac{1}{3} D^{2}\right) \bar{I}_{s s}+4 F\left(F-\frac{2}{3} D\right) \bar{I}_{u s}$ \\
\hline \hline
\end{tabular}

TABLE IV. Hairpin contributions from graphs involving octet baryons in the intermediate state. The coefficient $w_{i}^{(8,8)}$ is defined in eq. (45).

\begin{tabular}{ccc}
\hline \hline Baryon & $w_{i}^{(8,10)}$ & $w_{i}^{(10,10)}$ \\
\hline$p, n$ & 0 & 0 \\
$\Sigma^{+}$ & $\frac{4}{27}\left((F-D)\left(\bar{I}_{u s}^{\Delta}-\bar{I}_{s s}^{\Delta}\right)+2 F\left(\bar{I}_{u u}^{\Delta}-\bar{I}_{u s}^{\Delta}\right)\right)$ & $-\frac{2}{9} \mu_{\Sigma^{+}}^{\text {tree }}+\frac{20}{81} \mu_{C}$ \\
$\Sigma^{0}$ & $\frac{2}{27}\left((F-D)\left(\bar{I}_{u s}^{\Delta}-\bar{I}_{s s}^{\Delta}\right)+2 F\left(\bar{I}_{u u}^{\Delta}-\bar{I}_{u s}^{\Delta}\right)\right)$ & $-\frac{2}{9} \mu_{\Sigma^{0}}^{\text {tree }}$ \\
$\Sigma^{-}$ & 0 & $-\frac{2}{9} \mu_{\Sigma^{-}}^{\text {tree }}-\frac{20}{81} \mu_{C}$ \\
$\Lambda$ & $\frac{4}{27}\left((F-D)\left(\bar{I}_{u u}^{\Delta}-\bar{I}_{u s}^{\Delta}\right)+2 F\left(\bar{I}_{u s}^{\Delta}-\bar{I}_{s s}^{\Delta}\right)\right)$ & 0 \\
$\Xi^{0}$ & 0 & $-\frac{2}{9} \mu_{\Xi^{0}}^{\text {tree }}$ \\
$\Xi^{-}$ & 0 & $-\frac{2}{9} \mu_{\Xi^{-}}^{\text {tree }}-\frac{20}{81} \mu_{C}$ \\
$\Sigma^{0} \rightarrow \Lambda$ & $-\frac{1}{9} \mu_{\Sigma^{0} \Lambda}^{\text {tree }}$ \\
\hline \hline
\end{tabular}

TABLE V. Hairpin contributions from graphs involving both octet and decuplet baryons in the intermediate state. The coefficients $w_{i}^{(8,10)}$ and $w_{i}^{(10,10)}$ are defined in eq. (45).

have computed for the $w_{i}^{(r, s)}$ are shown in Table IV and Table $\mathrm{V}$.

Corrections of the form $\sim \sqrt{m_{q}}$ and $\sim m_{q} \log m_{q}$ have been computed in QCD and the

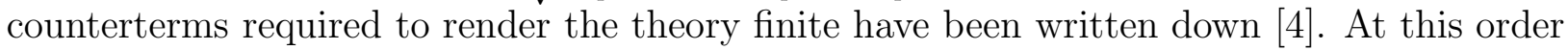
there is one relation between the magnetic moments,

$$
6 \mu_{\Lambda}+\mu_{\Sigma^{-}}-4 \sqrt{3} \mu_{\Sigma^{0} \Lambda}=4 \mu_{n}-\mu_{\Sigma^{+}}+4 \mu_{\Xi^{0}} .
$$

that is found to be satisfied at $\sim 5 \%$-level in nature. However, in QQCD we have been unable to find any $S U(3)$ relation between the magnetic moments when both the $\sim \sqrt{m_{q}}$ and $\sim M_{0}^{2} \log m_{q}$ contributions are considered. Of course, the trivial $S U(2)$ relation between the $\Sigma^{ \pm, 0}$ moments $\mu_{\Sigma^{+}}+\mu_{\Sigma^{-}}-2 \mu_{\Sigma^{0}}=0$ still holds.

\section{The Nucleon}

It is worth considering the chiral expansion of the nucleon magnetic moment explicitly. We have found that the magnetic moment of the proton is

$\mu_{p}=\left[\frac{\mu_{D}}{3}+\mu_{F}\right]\left[1-\frac{M_{0}^{2}(D-3 F)^{2}}{12 \pi^{2} f^{2}} \log \left(\frac{m_{\pi}^{2}}{\mu^{2}}\right)\right]-\frac{M_{N}}{24 \pi f^{2}}\left[8 D^{2} m_{\pi}+\mathcal{C}^{2} \mathcal{F}\left(m_{\pi}, \Delta, \mu\right)\right]$

where we have shown only the terms non-analytic in $m_{q}$. It has all the features we expect to find in QQCD. Kaon loops are absent as there are no strange quarks in the initial or final 
states. There are still pion loop contributions as the up and down quarks in the initial and final states can be routed through a meson-loop diagram without requiring the presence of a sea-quark. Further, if we insert the QCD values of the axial couplings [25], $F$ and $D$, we find that the pion loop contribution is somewhat smaller in QQCD than QCD. It will be interesting to learn the values of $F$ and $D$ in QQCD to see if this feature is actually present.

In order to gain some sort of understanding of how the QQCD extrapolation compares with the QCD extrapolation, we use the QCD values for the constants, that appear in eq. (47), $F=0.5, D=0.8$ [29], $|\mathcal{C}|=1.8$ [30], $f=132 \mathrm{MeV}$, and the physical value of the $\Delta-N$ mass splitting. Choosing to renormalize at the scale $\mu \sim 1 \mathrm{GeV}$, and keeping the kaon mass fixed at its physical value we vary the pion mass and determine the proton magnetic moment. The tree-level magnetic moment is fixed so that at one-loop it takes its experimental value for the physical pion mass, while $M_{0} \sim 750 \mathrm{MeV}$ is taken from the work in Ref. [32 (discussed in Ref. [18]). The magnetic moment of the proton verses the pion mass is shown in fig. 3. One sees that the extrapolation in QQCD is not so different to that

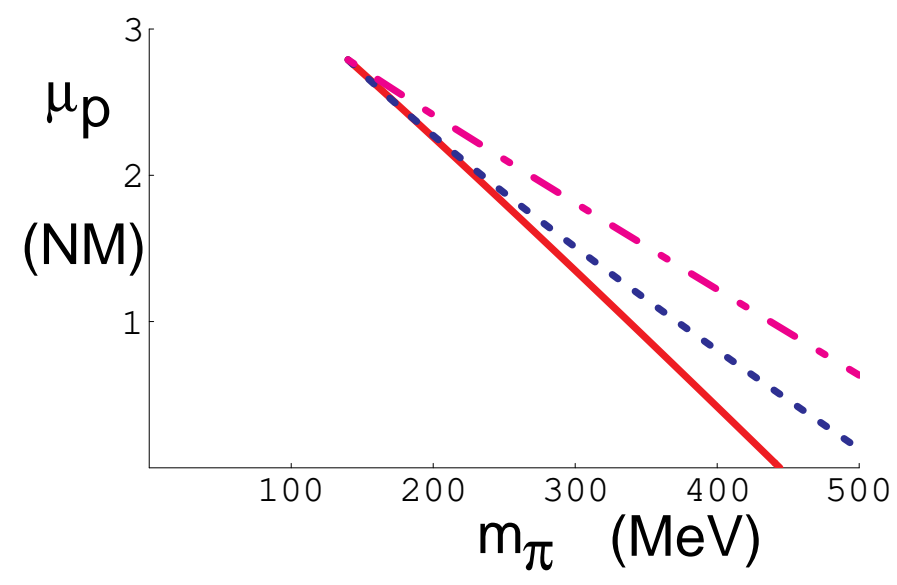

FIG. 3. The magnetic moment of the proton verses the pion mass, with the kaon mass fixed to its physical value. The solid curve corresponds to QCD with only the $\sim \sqrt{m_{q}}$ light-quark mass dependence included. The dashed curve corresponds to QQCD with $M_{0}=750 \mathrm{MeV}$ [39]. To demonstrate the sensitivity of the magnetic moment to $M_{0}$, the dot-dashed curve corresponds to $M_{0}=400 \mathrm{MeV}$ 33].

in QCD. The reduction in the $\sim \sqrt{m_{q}}$ terms is somewhat compensated by the contribution from the hairpin diagrams. If one uses the values of the axial couplings extracted at one-loop order, $F \sim 0.4, D \sim 0.6,|\mathcal{C}| \sim 1.4[30,31]$, the slope of each curve is reduced. It is clear from fig. 3 that the nucleon magnetic moment depends strongly upon $m_{q}$ at one-loop order (for these particular values of couplings), and that a higher order calculation in QQCD is likely required, as was the case in QCD [5]. Further, with these parameters, it would appear that an extrapolation from a pion mass of $\sim 250 \mathrm{MeV}$ or higher is unreliable without a higher order calculation. 


\section{CONCLUSIONS}

We have computed the contributions of the form $\sim M_{0}^{2} \log m_{q}$ and $\sim \sqrt{m_{q}}$ to the magnetic moments of the octet baryons in quenched chiral perturbation theory. The sickness of QQCD reveals itself yet again in the chiral expansion, with hairpin interactions providing the formally dominant correction near the chiral limit, giving a completely different chiral behavior than QCD. In performing this calculation we found it convenient to introduce ghost-baryon fields to describe the first, second and third floors of the $\mathbf{7 0}$ and $\mathbf{3 8}$ dimensional representations of $S U(3 \mid 3)$, the implementation of which we have detailed in the text.

In order to use our results, not only must the magnetic moments of the baryons be determined from the lattice, but also the quenched axial coupling constants f $F, D$ and $\mathcal{C}$, and the meson decay constant, $f$. Such calculations will enable an extrapolation of quenched lattice data from the lattice masses to the physical masses to make a prediction for the baryon magnetic moments in QQCD.

\section{ACKNOWLEDGMENTS}

I would like to thank Steve Sharpe for many useful discussions. Also, I would like to thank Aneesh Manohar and Jiunn-Wei Chen for useful comments on the manuscript. This work is supported in part by the U.S. Dept. of Energy under Grants No. DE-FG03-97ER4014.

\footnotetext{
${ }^{4}$ The quenched chiral corrections to these quantities have been previously computed 34.
} 


\section{REFERENCES}

[1] D. B. Leinweber, D. H. Lu and A. W. Thomas, Phys. Rev. D60, 034014 (1999); E. J. Hackett-Jones, D. B. Leinweber and A. W. Thomas, Phys. Lett. B489, 143 (2000).

[2] D. B. Leinweber, R. M. Woloshyn and T. Draper, Phys. Rev. D43, 1659 (1991).

[3] D. G. Caldi and H. Pagels, Phys. Rev. D10, 3739 (1974).

[4] E. Jenkins, M. Luke, A. V. Manohar and M. J. Savage, Phys. Lett. B302, 482 (1993); B388, 866 (1996)(E).

[5] U. -G. Meissner and S. Steininger Nucl. Phys. B499, 349 (1997).

[6] L. Durand and P. Ha, Phys. Rev. D58, 013010 (1998).

[7] S. J. Puglia and M.J. Ramsey-Musolf, Phys. Rev. D62, 034010 (2000).

[8] S. R. Sharpe, Nucl. Phys. B17 (Proc. Suppl.), 146 (1990).

[9] S. R. Sharpe, Phys. Rev. D46, 3146 (1992).

[10] C. Bernard and M. F. L. Golterman, Phys. Rev. D46, 853 (1992).

[11] J. N. Labrenz and S. R. Sharpe, Phys. Rev. D54, 4595 (1996).

[12] G. Chiladze, Phys. Rev. D57, 5586, (1998).

[13] P. H. Damgaard, J. C. Osborn, D. Toublan and J. J. M. Verbaarschot, Nucl. Phys. B547, 305 (1999).

[14] S. R. Sharpe and N. Shoresh, hep-lat/0108003.

[15] T. Guhr, J. Math. Phys. 34, 2541 (1993).

[16] S. R. Sharpe, Phys. Rev. D41, 3233 (1990);

[17] G. Colangelo and E Pallante, Nucl. Phys. B520, 433 (1998).

[18] M. F. L. Golterman, Acta Phys. Polon. B25, 1731 (1994).

[19] A. B. Balantekin, I. Bars and F. Iachello, Phys. Rev. Lett. 47, 19 (1981).

[20] A. B. Balantekin and I. Bars, J. Math. Phys. 23, 1239 (1981); J. Math. Phys. 22, 1810 (1981); J. Math. Phys. 22, 1149 (1981).

[21] J. -P. Hurni and B. Morel, J. Math. Phys. 24, 157 (1983).

[22] E. Jenkins and A. V. Manohar, Phys. Lett. B255, 558 (1991).

[23] E. Jenkins and A. V. Manohar, Phys. Lett. B259, 353 (1991).

[24] E. Jenkins, Nucl. Phys. B368, 190 (1992).

[25] E. Jenkins and A. V. Manohar, Baryon Chiral Perturbation Theory, talks presented at the workshop on Effective Field Theories of the Standard Model, Dobogoko, Hungary (1991);

[26] For a recent review see U.-G. Meißner, Essay for the Festschrift in honor of Boris Ioffe, in "Encyclopedia of Analytic QCD", edited by M. Shifman, to be published by World Scientific. hep-ph/0007092.

[27] S. Coleman and S. L. Glashow, Phys. Rev. Lett. 6, 423 (1961).

[28] C. K. Chow, Phys. Rev. D57, 6762 (1998).

[29] R. Flores-Mendieta, E. Jenkins and A. V. Manohar, Phys. Rev. D58, 094028 (1998).

[30] M. N. Butler, M. J. Savage and R. P. Springer, Nucl. Phys. B399, 69 (1993).

[31] M. J. Savage and J. Walden, Phys. Rev. D55, 5376 (1997).

[32] Y. Kuramashi, M. Fukugita, H. Mino, M. Okawa and A. Ukawa, Phys. Rev. Lett. 72, 3448 (1994).

[33] W. Bardeen, A. Duncan, E. Eichten, N. Isgur and H. Thacker, hep-lat/0106008.

[34] M. Kim and S. Kim, Phys. Rev. D58, 074509 (1998). 